\title{
Prognostic implication of the coronary microvascular dysfunction in patients with isolated left bundle branch block
}

Francisco J. Rodríguez Rodrigo, Juan Medina Peralta, Eddy Velásquez Arias, Ana Alegría Barrero, Teresa San Agustín Lascorz, Elena Pérez Pereira, Adriana Rodríguez Chaverri

Servicio Cardiología, Hospital Universitario Madrid Montepríncipe, Madrid, Spain

Email: frguezrodrigo@hotmail.com

Received 25 November 2013; revised 29 December 2013; accepted 7 January 2014

Copyright (C) 2014 Francisco J. Rodríguez Rodrigo et al. This is an open access article distributed under the Creative Commons Attribution License, which permits unrestricted use, distribution, and reproduction in any medium, provided the original work is properly cited. In accordance of the Creative Commons Attribution License all Copyrights (C) 2014 are reserved for SCIRP and the owner of the intellectual property Francisco J. Rodríguez Rodrigo et al. All Copyright (C) 2014 are guarded by law and by SCIRP as a guardian.

\section{ABSTRACT}

The present study aims to determine the influence of microvascular dysfunction (MVD) in the prognosis of patients presenting isolated left bundle branch block (LBBB). Methods: We studied 30 patients (pts), 22 males, 8 females, mean age $57 \pm 4$ years, with isolated LBBB, with a mean follow up of $48 \pm 6$ months. The control group consisted of 20 healthy individuals, 12 males, mean age $52 \pm 10$ years. Both groups were screened for cardiovascular risk factors (RF); they also had an echocardiogram and Coronary CT Scan, ruling out both structural heart disease and obstructive lesions of the epicardial coronary arteries. A myocardial perfusion study was then performed, with two groups emerging according to these results: Group A, 8 pts (26\%), with reversible perfusion defects, in which the diagnosis of MVD was suspected, and Group B, 22 pts (74\%), with either normal perfusion or minor septal/apical reversible defects (related to $\mathrm{LBBB}$ ). All Group A pts, and 9 of the Group B pts, underwent coronary arteriography, with intracoronary acetylcholine and nitroglycerine infusion, thus evaluating vasomotor response as endothelium dependent (acetylcholine) or endothelium independent (nitroglycerine). During follow up, we reviewed functional class, 12 lead ECG and echocardiogram on a yearly basis. Results: All Group A patients had an abnormal acetylcholine response; only three of them had abnormal response to nitroglycerine infusion, suggesting endothelium dependent MVD. Of those in Group B, only one patient had abnormal acetylcholine response. At the end of the follow up period, 3 pts (37\%) in Group A, showed functional class decrease vs 5 pts (22\%) of those in Group B. In Group A, a significant increase of End Diastolic Left Ventricle Diameter (EDLVD) was found (51.6 \pm 3.6 vs $59.3 \pm 6.8 \mathrm{~mm}$; p $<0.05)$ with significant decrease in LVEF (62 \pm 4.8 vs $46 \% \pm 3.7 \%$, p $<0.01$ ); both controls and Group $B$ showed no variation. In neither group major complications (death, heart failure admissions) were found. Conclusion: We confirm the association between MVD and a worse clinical prognosis in isolated LBBB patients. Repeated ischemia and myocardial fibrosis are highlighted as possible physiopathological mechanisms, precluding a progressive left ventricular function decrease, with a higher mortality and arrhythmia risk. Endothelial function preserving strategies, both preventive and therapeutic, might be useful in improving LBBB with MVD patient's prognosis.

\section{KEYWORDS}

Microvascular Dysfunction; Isolated Left Bundle Branch Block; Left Ventricular Dysfunction

\section{INTRODUCTION}

LBBB is usually associated with previous heart disease, but in up to $12 \%$ of cases, it appears not to be so, thus being accepted as isolated LBBB [1]. LBBB entails an increase in cardiovascular related death risk [2]. Higher than expected rates of high grade atrioventricular heart block and sudden death have been reported, and in coronary artery disease patients, a more extensive affection of the epicardial arteries, a more severe ventricular dysfunction and a lower survival rate have been described [3]. 
Previous studies regarding prognostic value of isolated LBBB yielded unhomogeneous results, due to different populations and study designs. Usually considered as a benign condition, in some cases, a progressive deterioration of the left ventricular function is noted, with a higher than expected mortality; the causal mechanisms and prognostic factors remain unknown.

Among those causes possibly related with a poor outcome, MVD was pointed out a few years back [4]. This hypothesis was highlighted after the X Syndrome (angor pectoris with normal coronary arteries) description; this was first suggested by Olser in 1910, in a patient with a long period recurrent angina with normal coronary arteries in necropsy [5]. A progressive deterioration of left ventricular contractility and, subsequently, a worse prognosis were described in some patients with either permanent LBBB or with frequency dependent LBBB [6]. Previous studies seem to underline an alteration of the coronary artery dilation capability (MVD) as a main factor in this poor outcome $[7,8]$.

This paper aims to investigate the correlation between coronary arteries micro vascular dysfunction and a worse clinical prognosis in patients with isolated LBBB who develop left ventricular dysfunction during follow up.

\section{METHODS}

The study group included 30 patients (22 male, 8 female), with a mean age of $57 \pm 4$ years, with isolated LBBB diagnosed in the six months before inclusion, with a mean follow up period of $48 \pm 6$ months. Structural heart disease was ruled out by echocardiography, and major epicardial coronary arteries were studied by means of Coronary CT Scan. The control group consisted in 20 normal subjects without LBBB (12 male, 8 female, $52 \pm$ 10 years) (Table 1 ).

The exclusion criteria were pregnancy, previous heart disease (congenital, valvular, ischemic or hypertensive cardiopathies), primary cardiomyopathies and connective tissue disorders. No previous history of pacemaker, atrial fibrillation and heart surgery was found both in patients and control subjects. Patients undergoing therapy susceptible of depressing heart contractility or affecting the conduction system were also excluded. Inclusion and exclusion criteria are summarized in Table 2.

We screened for major cardiovascular risk factors, specifically smoking history, high blood pressure, type 2 diabetes and dyslipidemias. All patients in the study group were in sinus rhythm, with a LBBB pattern in the ECG with a QRS complex duration $\geq 140 \mathrm{~ms}$ in men and $\geq 130$ $\mathrm{ms}$ in women, QS or rS in V1 and V2 precordial leads, and end QRS slurring in $\geq 2$ leads (V1, V2, V5, V6, I and aVl).

The study was approved by our institution ethics committee, and informed consent was obtained in all patients.

\subsection{Coronary CT Scan}

A coronary CT scan was performed as a means to rule

Table 1. Clinical data.

\begin{tabular}{cccc}
\hline & Healthy volunteers & LBBB patients & p \\
\hline Age & $52 \pm 10$ & $57 \pm 4$ & 0.09 \\
Sex & & & \\
Male & 12 & 22 & \\
Female & 8 & 8 & $<0.001$ \\
QRS duration & $99 \pm 10$ & $154 \pm 33$ & \\
\hline
\end{tabular}

Table 2. Exclusion and inclusion criterias.

\begin{tabular}{lll}
\hline & - & Both sexes. \\
Admission criteria & - & Age $18-65$ years. \\
& - & Left Bundle Branch Block diagnosed the previous six months. \\
& - & Sinus rhythm. \\
& - & Normal epicardial coronary arteries (Coronary CT Scan) \\
& - & No current structural cardiopathy (Echocardiogram). \\
\hline Exclusion criteria & - & Pregnancy. \\
& - & Congenital heart disease. \\
& - & Valvular heart disease. \\
& - & Epicardial coronary arteries lesions. \\
& - & Other cause myocardiopathies. \\
& - & Connective tissue disorders. \\
& - & Active antineoplasic therapy \\
& - & Therapy susceptible of depressing heart contractility or affecting the conduction system. \\
& - & Atrial fibrillation. \\
& - & Pacemaker \\
& &
\end{tabular}


out epicardial coronary artery stenosis. The study was obtained with a 64 slice tomographic scanner, with a temporal resolution of $165 \mathrm{~ms}$ and a spatial resolution of $0.4 \mathrm{~mm}^{3}$ (Siemens). When heart rate was higher than 65 bpm, intravenous metoprolol (5 $\mathrm{mg}$ up to a maximum of $10 \mathrm{mg}$ ) was administered. With antecubital venous access, 82 - $105 \mathrm{ml}$ of non-ionic contrast (Iodixanol) was then infused, followed by $50 \mathrm{ml}$ saline infusion at a $5 \mathrm{ml}$ per second rate.

\subsection{Echocardiographic Study}

Transthoracic echocardiographic studies were performed with commercially available ultrasound machines (Sonos 5000, Philips Ultrasound; Vivid System 4, GE/Vingmed), equipped with a multifrequency phased-array sector scan $3 \mathrm{mHz}$ probe. $\mathrm{M}$ mode measurements (in $\mathrm{mm}$ ) of the left ventricular walls, end diastolic diameter (EDD) and end systolic diameter (ESD) were obtained according to standardized protocols. 2D mode protocol included end diastolic volume index (EDVI, $\mathrm{ml} / \mathrm{m}^{2}$ ), end systolic volume index (ESVI, $\mathrm{ml} / \mathrm{m}^{2}$ ) and left ventricular ejection fraction (LVEF, \%) measurements as per Simpson’s method.

\subsection{Gammagraphic Study}

A Bruce protocol stress test was performed (CASE 16, GE Healthcare tread mill), with continuous ECG and blood pressure monitoring. Electrocardiographic response was considered non evaluable due to LBBB. Functional class was measured in METs.

At the final stage $27 \mathrm{mCi}(1.000 \mathrm{MBq})$ of $99 \mathrm{~m}$ Tcsestamibi were infused, and single photonic emission computerized tomography (SPECT) (Siemens) was obtained both immediately and in a 6 - 8 hours interval from injection of the isotope. A multiple spindle gamma camera, with high resolution and low energy collimators, acquired cardiac perfusion images. A $64 \times 64$ matrix with 40 s per image acquisition time were used.

Trans-axial slices reconstruction was performed with a specific dedicated computer program. Polar maps ("bull's eye") of the relative distribution in the left ventricle were rendered by using a volumetric sampling tool. Each polar map was normalized to its individual maximum. SPECT polar maps quantitative analysis allowed the calculation of the activity profile in every single short axis slice, from apex to base. The global values of each slice were represented in a color scale proportional to the activity, the center of this representation being occupied by the ventricular apex and the periphery by the basal segments. Contractility and systolic thickening of the left ventricle were evaluated employing ECG synchronized (gating) tomographic study. Fat oral intake was recommended to favor hepatobiliar radio tracer elimination, thus decreasing interference with the image acquisition.
According to this gammagraphic study, two groups were established:

Group A: 8 people (26\%), with perfusion reversible defects, either single or multiple, in which micro vascular dysfunction was suspected (Figure 1).

Group B: 22 people (74\%), with either normal perfusion tests or with minor isolated septal or apical defects, considered secondary to phasic coronary flow alteration induced by interventricular septum paradoxical movement, in itself secondary to LBBB.

\subsection{Endothelial Function Study}

All patients in Group A, and 9 patients in Group B, underwent coronary arteriography with intra coronary acetylcholine and nitroglycerine infusion as per usual protocol, in order to evaluate vasomotor response, either endothelium dependent or independent respectively. All vaso-active medications were stopped 48 hours before angiography. In all procedures femoral artery access was granted. An 8 Fr angioplasty guide catheter was placed in the left coronary artery ostium. Through this guide catheter, an infusion catheter (Cordis 3/2,5 Fr) was advanced over an angioplasty guide wire until it was placed in the proximal artery segment subject of study (left anterior descending in all cases). To avoid vasospasm induction, the guide wire was retrieved once the infusion catheter was properly placed.

Endothelial dependent vasodilatation response was analyzed in the first place by selective intracoronary acetylcholine infusion. Assuming an $80 \mathrm{ml} / \mathrm{m}$ coronary flow, we prepared $10^{6}, 10^{5}$ and $10^{4} \mathrm{~mol} / \mathrm{l}$ concentrations, estimating and intracoronary $10^{8}, 10^{7}$ and $10^{6} \mathrm{~mol} / \mathrm{l}$ acetylcholine concentrations. A basal 5\% glucose mechanical injection served as control, with further successively increasing concentration infusions of acetylcholine during two minutes $\left(10^{6}, 10^{5}\right.$ and $\left.10^{4} \mathrm{~mol} / \mathrm{l}\right)$. A manual injection coronariography was performed after each infusion.

Endothelial independent vasodilatation response was then studied, with $200 \mu \mathrm{g}$ nitroglycerine infusion and a further angiographic injection.

Blood pressure, heart rate end ECG were monitored continuously and registered immediately before each angiographic injection. For reproducibility allowances, during basal study the angiography angle, rotation, height and catheter type were registered.

\subsection{Quantitative Coronary Angiography}

For a better evaluation of the anterior descending artery a biplanar image equipment was used, acquiring orthogonal projections, with no need to modify radiological conditions between injections. The different angiographic projections were analyzed, post procedure, by the same 

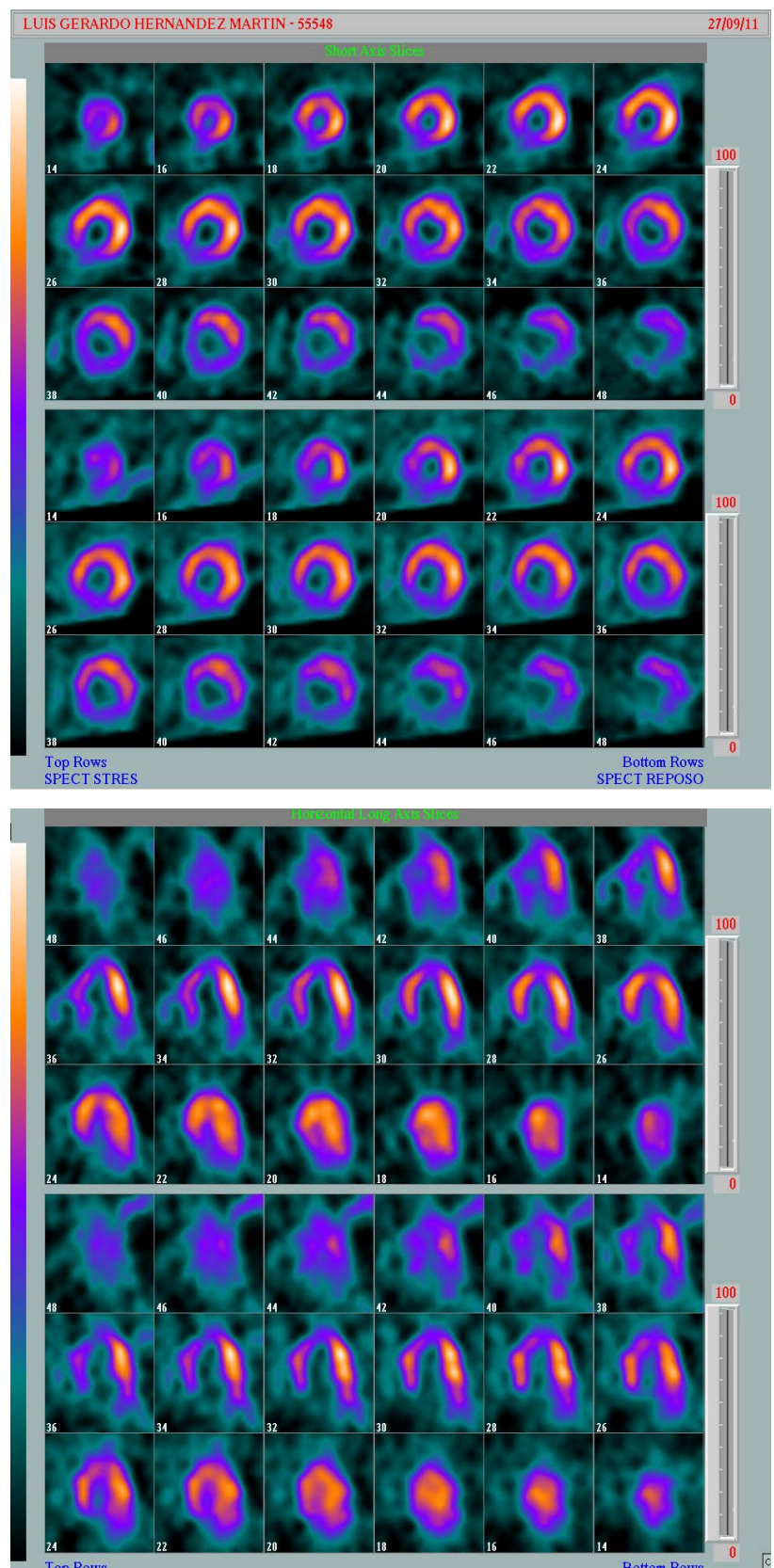

Figure 1. Septal and inferior defects (white arrow).

observer using automatic border detection software and the guide catheter as reference. For each acetylcholine dose, percentual luminal diameter variation from basal measurement was determined at three different arterial segments (proximal, medial and distal). In each patient the mean percentual variation in every segment was calculated for further analysis. Finally, mean percentual luminal diameter variation was measured between acetylcholine and nitroglycerine perfusion (after analyzing the two distal segments mean variation).

Intra observer method variability was analyzed by repetition of measurements in 20 angiographies after at
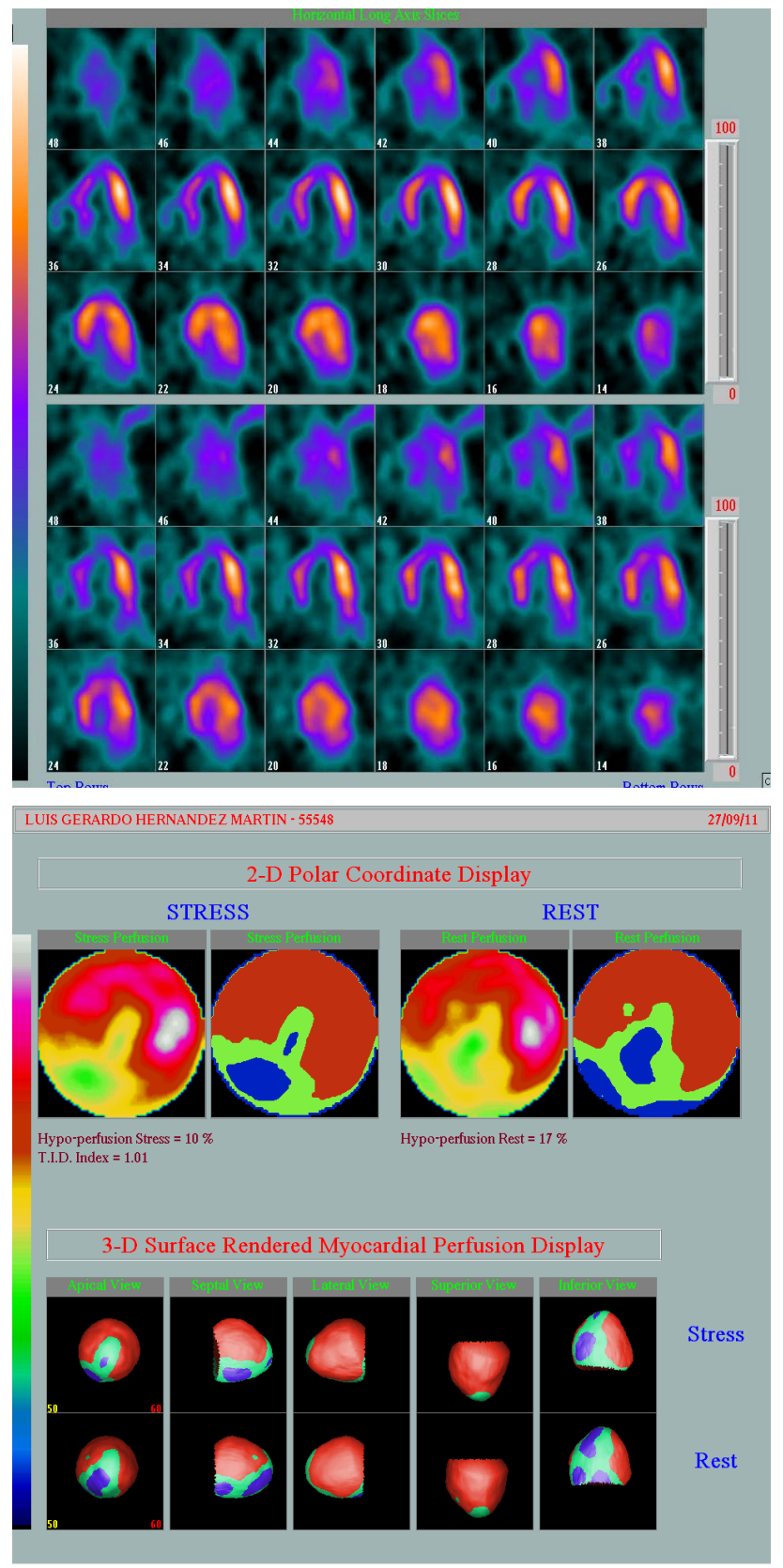

least 3 months delay (yielding a total number of 200 measurements). The observed differences (mean \pm 2 standard deviations) in the percentual luminal diameter variation were: $2.0 \% \pm 3.9 \%$ for basal values; $1.8 \% \pm 4.0 \%$ for the maximum acetylcholine dose and $1.7 \% \pm 3.8 \%$ after nitroglycerine.

The intra class correlation coefficients were 0.95 for basal values, 0.96 for the maximum acetylcholine dose and 0.98 after nitroglycerine. Thus, endothelial dysfunction was considered when a vasoconstriction after maximum acetylcholine dose higher than the analysis method variability (4\%) was found (Figure 2). 

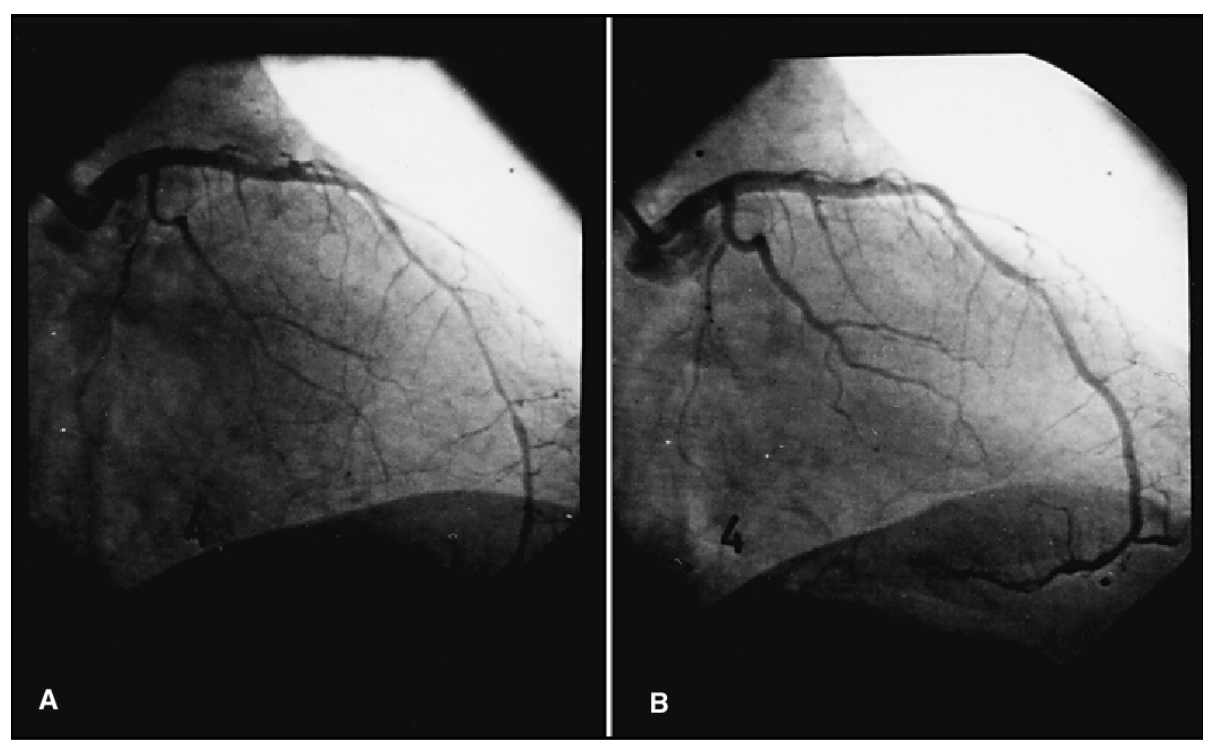

Figure 2. Abnormal vasomotor response in the anterior descending artery alter maximum dose acetylcholine infusion $\left(10^{-4} \mathrm{~mol} / \mathrm{l}\right)(\mathrm{A})$ with ulterior recovery of the luminal diameter $(\mathrm{B})$.

\subsection{Follow Up}

Patient mean follow up was of $48 \pm 6$ months. Functional class, echocardiogram and 12 lead ECG were performed on a yearly basis. At the end of follow up a coronary CT scan was obtained to rule out new coronary epicardial obstructive lesions in those patients with obvious ventricular function deterioration.

\subsection{Statistical Analysis}

Quantitative variables were expressed as mean \pm standard deviation. Paired data t Student method was used for quantitative variable comparison. Pearson correlation coefficient was used when determining relation between quantitative variables. A $\mathrm{p}<0.05$ value was considered statistically significant.

\section{RESULTS}

More Group A patients had more that 2 RF: 6 pts (75\%) vs 10 pts $(45 \%)(\mathrm{p}<0.05)$, while only 2 pts $(10 \%)$ in the control group showed this risk profile $(\mathrm{p}<0.001)$. Clinical data of the three groups are summarized in Table 3.

All patients were asymptomatic at inclusion (NYHA functional class I), with a left ventricle ejection fraction (LVEF) in the normal range and a slightly higher (though non significant) left ventricular end diastolic diameter (LVEDD) in group A pts, as summarized in Table 4.

To determine coronary vasomotor response to intracoronary acetylcholine infusion (endothelium mediated vasodilator response) and to nitroglycerine (non endothelium mediated vasodilator response) all Group A pts and 9 of the Group B pts underwent coronary arteriography. In Group A all 8 pts showed abnormal response to ace- tylcholine (arterial vasoconstriction) while only 3 of them had abnormal response to nitroglycerine infusion (no increase in luminal diameters); these results were compatible with microvascular endothelium dependent dysfunction. In Group B, just one patient showed abnormal response to acetylcholine while every patient in this group showed significant increase of the luminal diameter after intracoronary nitroglycerine infusion, as summarized in Table 5.

At one year follow up functional class, 12 lead ECG and echocardiographic measurements were recorded. No atrioventricular conduction defects or QRS duration increases were found. At the end of follow up, 3 pts (37\%) in Group A showed a 1 - 2 grades worsening of their functional class, while only 5 pts (22\%) of those in Group B. LVEDD showed significant increase (51.6 \pm 3.6 vs $59.3 \pm 6.8 \mathrm{~mm}$; $\mathrm{p}<0.05$ ) with a significant decrease in LVEF $62 \pm 4.8$ vs $46 \% \pm 3.7 \%, \mathrm{p}<0.01$ ) in Group A patients, while these values remained unaltered in both Group B pts and controls. No deaths or heart failure hospital admissions were recorded Table 6 .

Functional class deterioration, ejection fraction decrease, or both, were found in eleven patients (7 in group A, 4 in Group B). All of them had a new coronary CT scan, but no new epicardial coronary lesions were found.

\section{DISCUSSION}

Isolated bundle branch block, without apparent or suspected structural heart disease, has a $0.28 \%$ prevalence, with a significantly higher frequency of the right bundle block versus that of the left bundle $(0.18 \%$ vs $0.1 \%$ respectively, $\mathrm{p}<0.01$ ), and with a combined prevalence of both conduction defects increasing with age [8]. In our 
Table 3. Clínical profile of Group A, Group B and controls.

\begin{tabular}{cccccccc}
\hline & \multicolumn{5}{c}{ Cardiovascular risk factors (RF) } \\
\hline & Age & Sex & Smoker & Hyperthension & Diabetes & Dislipemia & $>2 \mathrm{RF}$ \\
\hline GROUP A (n = 8 patients) & $57 \pm 4$ & $6 \mathrm{M}, 2 \mathrm{~F}$ & 6 & 2 & 1 & 4 & $6^{*}$ \\
GROUP B n $=\mathbf{2 2}$ & $57 \pm 4$ & $16 \mathrm{M}, 6 \mathrm{~F}$ & 8 & 3 & 2 & 6 & $10^{*}$ \\
CONTROL $\mathbf{n}=\mathbf{2 0}$ & $52 \pm 10$ & $12 \mathrm{M}, 8 \mathrm{~F}$ & 3 & 0 & 0 & 4 & $2^{\dagger}$ \\
\hline
\end{tabular}

Group A, multiple perfusion defects; Group B, normal or isolated interventricular septum perfusion defect gammagraphy. Values expressed as mean \pm SD. Significant statistical difference: ${ }^{*} \mathrm{p}<0.05,{ }^{\dagger} \mathrm{p}<0.001$.

Table 4. Functional class and echocardiopgraphic measurements at admission.

\begin{tabular}{|c|c|c|c|c|c|c|}
\hline & FC I & EDD (mm) & $\operatorname{EDVI}\left(\mathrm{ml} / \mathrm{m}^{2}\right)$ & ESD (mm) & $\operatorname{ESVI}\left(\mathbf{m l} / \mathbf{m}^{2}\right)$ & EF (\%) \\
\hline GROUP A ( $(\mathrm{n}=8$ patients) & 8 & $51.6 \pm 3.6$ & $75 \pm 22.8$ & $23 \pm 0.8$ & $22 \pm 8.1$ & $62 \pm 4.8$ \\
\hline GROUP B (n = 22 patients) & 22 & $51.0 \pm 3.3$ & $74 \pm 19.3$ & $22 \pm 0.7$ & $21 \pm 11.3$ & $64 \pm 6.8$ \\
\hline HEALTHY CONTROLS ( $n=20$ patients) & 20 & $51.1 \pm 3.2$ & $74 \pm 14.2$ & $21 \pm 0.8$ & $21 \pm 10.6$ & $67 \pm 6.2$ \\
\hline
\end{tabular}

FC, functional class; EDD, end diastolic diameter; EDVI end diastolic volume index; ESD, end systolic diameter; ESDVI end systolic volume index; EF, left ventricular ejection fraction. Significant statistical difference: ${ }^{*} \mathrm{p}<0.05,{ }^{\dagger} \mathrm{p}<0.001$.

Table 5. Arterial vasomotor response to acetylcholine and nitroglycerine.

\begin{tabular}{ccc}
\hline & Abnormal response to AC & Abnormal response to NTG \\
\hline Group A ( $=8$ patients) & 8 patients (100\%) & 3 patients $(37 \%)$ \\
Group B ( $=9$ patients) & 1 patient $(11 \%)$ & $0(0 \%)$ \\
\hline
\end{tabular}

AC: Acetylcholine. NTG: Nitroglycerine.

Table 6. Functional class and echocardiopgraphic measurements at end of follow up.

\begin{tabular}{|c|c|c|c|c|c|c|}
\hline & FC I (NYHA) & EDD(mm) & $\operatorname{EDVI}\left(\mathrm{ml} / \mathbf{m}^{2}\right)$ & ESD (mm) & $\operatorname{ESVI}\left(\mathrm{ml} / \mathbf{m}^{2}\right)$ & EF (\%) \\
\hline GROUPA ( $\mathrm{n}=8$ patients) & $5(62 \%)$ & $59.3 \pm 6.8^{*}$ & $84 \pm 25.3$ & $30 \pm 1.8$ & $28 \pm 9.2$ & $46 \pm 3.7^{*}$ \\
\hline GROUP B (n = 22 patients) & 17 (77\%) & $52.0 \pm 3.5$ & $75 \pm 18.3$ & $24 \pm 0.9$ & $22 \pm 10.2$ & $62 \pm 5.7$ \\
\hline HEALTHY CONTROLS ( $\mathrm{n}=20$ patients) & 20 & $52.1 \pm 2.2$ & $73 \pm 12.2$ & $23 \pm 1.2$ & $22 \pm 9.8$ & $66 \pm 5.2$ \\
\hline
\end{tabular}

FC, functional class; EDD, end diastolic diameter; EDVI end diastolic volume index ; ESD, end systolic diameter; ESDVI end systolic volume index; EF, left ventricular ejection fraction. Significant statistical difference: ${ }^{*} \mathrm{p}<0.05,{ }^{\dagger} \mathrm{p}<0.001$.

work we found a worse clinical outcome in isolated LBBB patients with associated MVD data.

Since Wiggers's first description [9], other studies suggested that an abnormal ventricular activation pattern caused by LBBB or right ventricular apical stimulation is linked to left ventricular function deterioration, structural changes and regional myocardial perfusion differences. An anomalous inverse activation sequence is found in LBBB patients, leading to interventricular asynchrony with interventricular septum anomalous movement and subsequently reducing septal contribution to left ventricular systolic performance [10]. Isolated LBBB related interventricular and intraventricular asynchrony by itself would not account for the divergent progression of left ventricular anatomical and functional parameters found in different subsets of patients.

Coronary microvascular dysfunction hypothesis as explanatory cause of the left ventricular function worsening found in isolated LBBB patients emerges after the $\mathrm{X}$ Syndrome (angor pectoris with normal coronary arteries) description. Cannon RO et al. [11] described a poor clini- cal progression with significant deterioration of the left ventricular contractility through the years in a subgroup of X Syndrome patients presenting with either permanent $\mathrm{LBBB}$ or rate dependant $\mathrm{LBBB}$. In that study, sings of coronary microvascular dysfunction were described in that subset of patients, mainly an abnormal vasodilator reserve as evidenced by the following findings: a) Coronary blood flow at rest was normal in the coronary arteriography. After dipyridamole infusion, maximal myocardial blood flow increase was half of that measured in a control group without LBBB. b) Lactate production increased during high rate atrial pacing, a well known finding in patients with obstructive coronary artery disease [12] and c) Significant lowering of the left ventricular contractile reserve, as measured with basal and post exercise isotopic ventriculography. Other studies confirm these findings, remarking myocardial ischemia as the underlying physiopathological mechanism even in the presence of normal epicardial coronary arteries, which may lead to latent cardiomyopathy with prognostic significance [6,13-16]. 
We studied 30 patients with isolated LBBB and normal epicardial coronary arteries. According to stress gammagraphy results, two patient groups were established: Group A, with 8 patients (26\%) with single or multiple reversible perfusion defects, in which MVD was accepted, and Group B (22 patients, 74\%) with either normal or with isolated septal defects, secondary to paradoxical movement of the interventricular septum related to the LBBB itself.

The clinical profile was homogenous in both groups but for a higher presence of RF in Group A.

Huge epidemiologic studies $[17,18]$ have defined those factors related to coronary disease and its complications. This RF may act precociously, causing vascular damage well before clinical disease becomes evident. In arteriosclerotic affected adults, endothelial dysfunction has been described $[19,20]$, but it is indeed a remarkable early event in atherogenesis [21,22]. The cause of the alteration of the endothelium dependent vasodilatation in risk factors prone subjects remains unclear, but decreased liberation or synthesis, or both, of the endothelial derived relaxing factor (RFED) have been strongly suggested [23-25] to be related. Zeiher et al. [26] showed an increased coronary blood flow after intracoronary acetylcholine infusion in a group of healthy subjects with no cardiovascular risk factors and normal epicardial coronary arteries. Those subjects with hypercholesterolemia and high plasmatic LDL concentrations, even with angiographycallly normal arteries, had a selective coronary dysfunction with vasoconstriction and decreasing coronary blood flow after acetylcholine infusion.

As expected, coronary vasomotor response to intracoronary acetylcholine infusion (endothelium derived vasodilatation) and to nitroglycerine (non endothelium dependent vasodilatation) diverged in both groups. All 8 group A patients showed an abnormal response to acetylcholine (arterial vasoconstriction) and only 3 of them an abnormal response to the nitroglycerine bolus (absence of any increase in the arterial diameter), which is compatible with endothelium dependent micro vascular dysfunction. Of those in Group B just one patient showed abnormal acetylcholine response while all of them reacted with intra arterial diameter significant increase after intracoronary nitroglycerine infusion, consistent with a preserved coronary reserve. Considering a definite relation being established between cardiovascular risk factors and microvascular dysfunction in the absence of epicardial coronary arteries stenosis, the additional effect of isolated LBBB on the progressive deterioration of the left ventricular function remains questionable.

All patients at inclusion presented a diskinetic movement of the interventricular septum as only sing of ventricular asynchrony, with well in the normal range LVEF and ventricular measurements. Patients were reviewed on a yearly basis, with current functional class assessment and new ECG and echocardiograms. Neither QRS complex prolongation nor atrioventricular conduction defects were observed.

At the end of the follow up period a greater functional class deterioration was found in Group A patient vs Group B (37\% vs 22\%). Compared to inclusion values, Group A patients showed an increase in end diastolic left ventricular diameter (EDLVD) (51.6 \pm 3.6 vs $59.3 \pm 6.8$ $\mathrm{mm} ; \mathrm{p}<0.05)$ and a LVEF drop (62 \pm 4.8 vs $46 \% \pm$ $3.7 \%, \mathrm{p}<0.01$ ) while both values remained unaltered in Group B. No deaths were recorded in any group.

The role of MVD in those LBBB patients who presented left ventricular function deterioration at follow up might be related to myocardial fibrosis. One study [27] describes SPECT images in 21 dilated cardiomyopathy patients, $10 \mathrm{~min}$ and 2 hours after basal $111 \mathrm{MBq}^{201} \mathrm{Tl}$ injections. Myocardial uptake patterns were related to endomyocardial biopsy results, and an inverse relation between isotopic uptake percentage and the fibrosis/ normal tissue ratio was found. Fibrosis found in the area of a focal perfusion defect is probably linked to intraventricular conduction delay, ventricular dyssynchrony and left ventricular systolic function deterioration. Left ventricular diastolic function might as well be affected through prolonged systolic intervals, asynchronous relaxation, and both metabolic and energetic deficits [28,29]. Myocardial fibrosis related focal left ventricular perfusion defects might be an arrythmogenic substrate with a risk for ventricular arrhythmias [30].

Group A patients in our study, with perfusion defects and microvascular dysfunction sings in the coronary arteriography, might show myocite destruction and repairing fibrosis, as some autopsy studies have shown both in humans [31,32] and experimental models [33,34] These fibrotic areas might be responsible of the functional class deterioration, the increase in LVEDD and the LVEF drop found in Group A pts, worsening the clinical prognosis compared to those in Group B.

In conclusion, the proposed mechanisms involved in the worse clinical evolution found in isolated $\mathrm{LBBB}$ and MVD patients are: A) LBBB induced inter and intra ventricular asynchrony and coronary phase flow alteration, which in turn lead to left ventricular function deterioration, end diastolic left ventricular pressure increase and subsequent subendocardial perfusion drop. B) The presence of cardiovascular risk factors would enable microvascular dysfunction, ongoing ischemia and myocardial fibrosis, leading to a left ventricular function progressive deterioration, with a higher mortality and arrhythmia risk (Figure 3). Early adoption of therapeutic and prevention strategies aimed to preserve the endothelial function in these patients with LBBB and microvascular dysfunction data, might prove useful in improving 


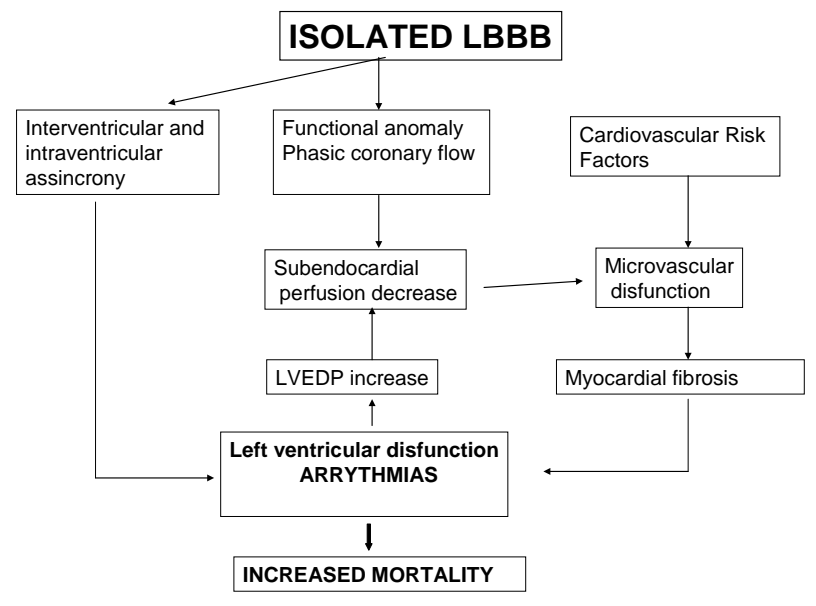

Figure 3. Physiological mechanisms involved.

their clinical progression.

\section{REFERENCES}

[1] Jain, A.C. and Mehta, M.C. (2003) Etiologies of left bundle branch block and correlations to hemodynamic and angiographic findings. American Heart Journal, 91, 13751382.

[2] Miller, W.L., Ballman, K.V., Hodge, D.O., et al. (2005) Risk factor implications of incidentally discovered uncomplicated bundle branch block. Mayo Clinic Proceedings, 80, 1585-1896.

http://dx.doi.org/10.4065/80.12.1585

[3] Eriksson, P., Wilhelmson, L. and Rosengren, A. (2005) Bundle-branch block in middle-Aged men: Risk of complications and death over 28 years. European Heart Journal, 26, 2300-2316.

http://dx.doi.org/10.1093/eurheartj/ehi580

[4] Opherk, D., Zebe, H., Weihe, E., Mall, G., Durr, C., Gravert, B., Mehmel, H.C., Schwarz, F. and Kubler, W. (1981) Reduced coronary dilatory capacity and ultrastructural changes of the myocardium in patients with angina pectoris but normal coronary arteriograms. Circulation, 63, 817-825.

http://dx.doi.org/10.1161/01.CIR.63.4.817

[5] Osler, W. (1910) The Lumleian Lectures on angina pectoris. Lancet, 1, 839. http://dx.doi.org/10.1016/S0140-6736(00)51244-6

[6] Cannon, R.O., Wattson, R.M., Rosing, D.R. and Epstein, S.E. (1983) Angina caused by reduced vasodilator reserve of the small coronary arteries. American College of Cardiology Foundation, 1, 1359-1373. http://dx.doi.org/10.1016/S0735-1097(83)80037-0

[7] Cannon, R.O., Bonow, R.O., Bacharach, S.L., Green, M.V., Rosing, D.R., Leon, M.B., Watson, R.M. and Epstein, S.E. (1985) Left ventricular dysfunction in patients with angina pectoris, normal epicardial coronary arteries and abnormal vasodilator reserve. Circulation, 71, 218226. http://dx.doi.org/10.1161/01.CIR.71.2.218

[8] Fahy, G.J., Pinski, S.L., Miller, D.P., et al. (1996) Natural history of isolated bundle branch block. American Journal of Cardiology, 77, 1185-1190. http://dx.doi.org/10.1016/S0002-9149(96)00160-9

[9] Wiggers, C.J. (1925) The muscular reactions of the mammaliam ventricles to artificial surface stimuli. American Journal of Physiology, 73, 346-378.

[10] Grines, C.L., Bashore, T.M., Boudoulas, H., et al. (1989) Functional abnormalities in isolated left bundle branch block. The effect of interventricular asynchrony. Circulation, 79, 845-853.

http://dx.doi.org/10.1161/01.CIR.79.4.845

[11] Cannon, R.O., Bonow, R.O., Bacharach, S.L., Green, M.V., Rosing, D.R., Leon, M.B., Watson, R.M. and Epstein, S.E. (1985) Left ventricular dysfunction in patients with angina pectoris, normal epicardial coronary arteries and abnormal vasodilator reserve. Circulation, 71, 218226. http://dx.doi.org/10.1161/01.CIR.71.2.218

[12] Cannon, R.O., Wattson, R.M., Rosing, D.R. and Epstein, S.E. (1983) Angina caused by reduced vasodilator reserve of the small coronary arteries. Journal of the American College of Cardiology, 1, 1359-1373. http://dx.doi.org/10.1016/S0735-1097(83)80037-0

[13] Cannon, R.O., Schenke, W.H., Leon, M.B., Rosing, D.R., Urquart, J. and Epstein, S.E. (1987) Limited coronary flow reserve after dipyridamole in patients with ergonovine-induced coronary vasoconstriction. Circulation, 75, 163-170. http://dx.doi.org/10.1161/01.CIR.75.1.163

[14] Greenberg, M.A., Grose, R.M., Strain, J.E., McGuinis, J. and Cohen, M.V. (1984) Decreased coronary dilatory response in syndrome X (abstract). Circulation, 70, II-21.

[15] Legrand, V., Hodgson, J.M., Bates, E.R., Aueron, F.M., Mancini, G.B.J., Smith, J.S., Gross, M.D. and Vogel, R.A. (1985) Abnormal coronary flow reserve and abnormal radionuclide exercise tests in patients with normal coronary angiograms. Journal of the American College of Cardiology, 6, 1245-1251. http://dx.doi.org/10.1016/S0735-1097(85)80209-6

[16] Schmidt, D.H., Hendrix, L., Lassar, T., Ray, G. and Port, S. (1984) Ergonovine/dipyridamole-induced changes in regional myocardial perfusion in patients with angina and normal coronary arteries (abstract). Circulation, 70, II274.

[17] Castelli, W.P. (1984) Epidemiology of coronary heart disease. The framingham study. American Journal of Medicine, 76, 4-12. http://dx.doi.org/10.1016/0002-9343(84)90952-5

[18] Lipid Research Clinics Program. (1984) The Lipid Research Clinics Primary Prevention Trial results. Journal of the American Medical Association, 251, 351-364. http://dx.doi.org/10.1001/jama.1984.03340270029025

[19] Ludmer, P.L., Selwyn, A.P., Shook, T.L., et al. (1986) Paradoxical vasoconstriction induced by acetylcholine in atherosclerotic coronary arteries. The New England Journal of Medicine, 315, 1046-1051. http://dx.doi.org/10.1056/NEJM198610233151702

[20] Nabel, E.G., Selwyn, A.P. and Ganz, P. (1990) Large coronary arteries in humans are responsive to changing blood flow an endothelium-dependent mechanism thal fails in patients with atherosclerosis. Journal of the Ame- 
rican College of Cardiology, 16, 349-356. http://dx.doi.org/10.1016/0735-1097(90)90584-C

[21] Ross, R. (1986) The pathogenesis of atherosclerotic-An update. The New England Journal of Medicine, 8, 488500. http://dx.doi.org/10.1056/NEJM198602203140806

[22] Celermajer, D.S., Sorensen, K.E., Gooch, V.M., et al. (1992) Non-invasive detection of endothelial dysfunction in children and adults at risk of atherosclerosis. Lancet, 340, 1111-1115. http://dx.doi.org/10.1016/0140-6736(92)93147-F

[23] Verbeuren, T.J., Jordaens, F.H., Zonuckeyn, L.I., Van Howe, C.E. and Herman, A.O. (1986) Effect of hypercholesterolemia on vascular reactivity in the rabbit I. Endothelium-dependent and endothelium independent contractions and relaxations in isolated arteries o f control and hypercholesterolemic rabbits. Circulation Research, 58, 552-564. http://dx.doi.org/10.1161/01.RES.58.4.552

[24] Bossaller, C., Habbib, G.B., Yamamoto, H., Williams, C., Wells, S. and Henry, P.D. (1987) Impaired muscarinic endothelium-dependent relaxation and cyclic guanosine 5'-monophosphate formation in atherosclerotic human coronary artery and rabbit aorts. The Journal of Clinical Investigation, 79, 170-174. http://dx.doi.org/10.1172/JCI112779

[25] Egashira, K., Inco, T., Hirooka, Y., et al. (1993) Effects of age on endothelium-dependent vasodilation of resistance coronary artery by acethylcholine in humans. Circulation, 88, 77-81. http://dx.doi.org/10.1161/01.CIR.88.1.77

[26] Zeiher, A.M., Drexler, H., Wollschlager, H., Just, H. (1991) Modulation of coronary vasomotor tone in humans. Progressive endothelial dysfunction with different early stages of coronary atherosclerosis. Circulation, 83, 391-401.

http://dx.doi.org/10.1161/01.CIR.83.2.391

[27] Watanabe, M., Gotoh, K., Nagashima, K., Uno, Y., Noda, T., Nishigaki, K., Takemura, G., Kanoh, M., Yasuda, N., Ohno, Y., Minatoguchi, S. and Fujiwara, H. (2001) Relationship between thallium-201 myocardial SPECT and findings of endomyocardial biopsy specimens in dilated cardiomyopathy. Annals of Nuclear Medicine, 15, 13-19.

\section{ACRONYMS}

ECG: Electrocardiogram.

CT: Computerized Axial Tomography.

LBBB: Left bundle branch block.

MVD: Coronary micro vascular dysfunction.

LVEDD: Left ventricle end diastolic diameter (mm).

LVESD: Left ventricle end systolic diameter (mm).

EDVI: End diastolic volume index $\left(\mathrm{ml} / \mathrm{m}^{2}\right)$.
http://dx.doi.org/10.1007/BF03012125

[28] Nowak, B., Sinha, A.M., Schaefer, W.M., Koch, K.C., Kaiser, H.J., Hanrath, P., Buell, U. and Stellbrink, C. (2003) Cardiac resynchronization therapy homogenizes myocardial glucose metabolism and perfusion in dilated cardiomyopathy and left bundle branch block. Journal of the American College of Cardiology, 41, 1523-1528. http://dx.doi.org/10.1016/S0735-1097(03)00257-2

[29] Sundell, J., Engblom, E., Koistinen, J., Ylitalo, A., Naum, A., Stolen, K.Q., Kalliokoski, R., Nekolla, S.G., Airaksinen, K.E., Bax, J.J. and Knuuti, J. (2004) The effects of cardiac resynchronization therapy on left ventricular function, myocardial energetics, and metabolic reserve in patients with dilated cardiomyopathy and heart failure. Journal of the American College of Cardiology, 43, 1027-1033. http://dx.doi.org/10.1016/j.jacc.2003.10.044

[30] Soejima, K., Stevenson, W.G., Sapp, J.L., Selwyn, A.P., Couper, G. and Epstein, L.M. (2004) Endocardial and epicardial radiofrequency ablation of ventricular tachycardia associated with dilated cardiomyopathy: The importance of low-voltage scars. Journal of the American College of Cardiology, 43, 1834-1842. http://dx.doi.org/10.1016/j.jacc.2004.01.029

[31] Torres, C.M. (1958) Arteriosclerosis of the fine arterial branches of the myocardium (Chagas coronaritis ) and focal myocytolysis in chronic Chagas'heart disease. Hospital, 54, 19-34.

[32] Koberle, F. (1974) Pathogenesis of Chagas'disease. Ciba Foundation symposium, 20, 137-158.

[33] Rossi, M.A., Gonzalves, S. and Ribeiro-dos-Santos, R. (1984) experimental Tripanosoma cruzi cardiomyopathy in BALB/c mice: The potencial role of intravascular platelet aggregation in its genesis. American Journal of Pathology, 114, 209-216.

[34] Morris, S.A., Weiss, L.M., Factor, S., Bilezikian, J.P., Tanowitz, H. and Wittner, M. (1989) Verapamil ameliorates clinical, pathologic and biochemical manifestations of experimental chagasic cardiomyopathy in mice. Journal of the American College of Cardiology, 14, 782789. http://dx.doi.org/10.1016/0735-1097(89)90126-5

ESVI: End systolic volume index $\left(\mathrm{ml} / \mathrm{m}^{2}\right)$.

LVEF: Left ventricle ejection fraction (\%).

RF: Risk factors.

SPECT: Single photon emission computerized tomography.

EDRF: Endothelial derived relaxing factor.

NO: Nitric oxide.

CMRI: Cardiac magnetic resonance imaging. 